\title{
Validação do teste de inibição pelo ácido aminofenilborônico para triagem de Klebsiella pneumoniae carbapenemases (KPC)
}

Primeira submissão em 21/11/11 Última submissão em 04/10/12 Aceito para publicação em 13/11/12 Publicado em 20/12/12

\section{Validation of inhibition test by aminophenyl boronic acid to Klebsiella pneumoniae carbapenemases (KPC) screening}

Claudia Michele de Araujo Borba'; Viviane Matoso de Oliveira²; Lavínia N. V. S. Arend'; Marcelo Pilonetto

\section{unitermos}

Ácido aminofenil borônico

Klebsiella pneumoniae

Carbapenemase

KPC

\section{resumo}

Introdução: A produção de enzimas Klebsiella pneumoniae carbapenemase (KPC) tem se tornado um importante e preocupante mecanismo de resistência, e ensaios que combinem alta sensibilidade e alta especificidade para a detecção dessas enzimas são escassos. Objetivo: Validar o teste de inibição pelo ácido 3-aminofenilborônico como método de triagem fenotípica de cepas produtoras de enzima KPC, comparando os resultados obtidos com os de testes confirmatórios por reação em cadeia da polimerase (PCR). Metodologia: Avaliou-se o uso do ácido 3-aminofenilborônico impregnado em discos de antibióticos de imipenem, meropenem e ertapenem. Foram testadas 36 cepas positivas e 12 negativas, todas confirmadas por PCR. Foram ainda testadas três concentrações diferentes de ácido borônico: 300, 400 e $600 \mu \mathrm{g}$. Resultados: Entre as cepas positivas testadas, o resultado mais adequado se deu com a adição do composto em disco contendo ertapenem, com especificidade de 100\%, porém com sensibilidade de apenas 50\%. Conclusão: Novos estudos são necessários, sobretudo no que diz respeito à padronização da técnica e aos insumos utilizados, pois o método se revela promissor na triagem de cepas produtoras de KPC.

\section{abstract}

Introduction: The production of Klebsiella pneumoniae carbapenemases enzymes (KPC) has become an important and worrisome resistance mechanism. Furthermore, tests that combine high sensitivity and high specificity for the detection of these enzymes are scarce. Objective: To validate the inhibition test by 3-aminophenyl boronic acid as a phenotypic screening method for KPC-producing strains by comparing the results with confirmatory polymerase chain reaction testing (PCR). Methods: We evaluated the use of 3-aminophenyl boronic acid applied on disks with imipenem, meropenem and ertapenem antibiotics. 36 strains were positive and 12 were negative, all confirmed by $P C R$. Three different concentrations of boronic acid were also tested: 300, 400 and $600 \mu \mathrm{g}$. Results: Among the positive strains, the results were more accurate with the addition of the compound to the ertapenem disk, presenting $100 \%$ specificity and $50 \%$ sensitivity. Conclusion: Further studies are required, mainly regarding the standardization of the technique and materials, since the method seems to be promising as to the screening of KPC strains. key words

Aminophenyl boronic acid

Klebsiella pneumonia

Carbapenemases

KPC

1. Especialista em Microbiologia; farmacêutica bioquímica.

2. Mestra em Ciências Farmacêuticas; farmacêutica bioquímica.

3. Mestre em Ciências Farmacêuticas; farmacêutico bioquímico; professor de Microbiologia da Pontifícia Universidade Católica do Paraná (PUC-PR). 


\section{Introdução}

A produção de enzimas Klebsiella pneumoniae carbapenemase (KPC) tem se tornado um importante e preocupante mecanismo de resistência ${ }^{(1,14,15,20)}$. O grupo das carbapenemases tem alto potencial de disseminação, pois se localiza no plasmídio e, embora as KPC sejam predominantemente encontradas em $K$. pneumoniae, há relatos das enzimas em Enterobacter spp., Salmonella spp., Klebsiella oxytoca, Citrobacter

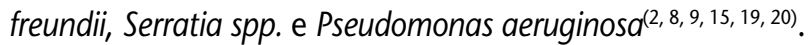

O composto 3-aminofenil ácido borônico (APBA) tem sido utilizado com sucesso na detecção de betalactamases classe $\mathrm{C}$ mediada por plasmídeo ${ }^{(4,17)}$. Esse composto é descrito como inibidor reversível das enzimas do tipo $\mathrm{KPC}^{(18)}$. O método foi descrito por Coudron ${ }^{(5)} \mathrm{em} 2005$ e consiste em um teste de disco combinado. A sinergia do ácido fenilborônico (PBA) com os antibióticos foi aplicada para a identificação fenotípica do primeiro isolado produtor de KPC na Grécia. Posteriormente, uma avaliação mais aprofundada, com uma grande coleção de isolados produtores de KPC, apresentou um claro efeito sinérgico entre o PBA e carbapenens, inferindo uma aparente interação da molécula de PBA com o sítio ativo das enzimas KPC de classe $A^{(13)}$. Estudos que combinaram imipenem, cefepime e meropenem com PBA mostraram alta sensibilidade e especificidade para detecção de KPC, cujo PBA aumentou de 8 a $11 \mathrm{~mm}$ o halo de inibição do meropenem para a maioria dos isolados KPC positivos ${ }^{(18)}$.

No entanto, ensaios que combinam alta sensibilidade e alta especificidade na detecção dessas enzimas não existem $^{(18)}$. Assim, é imprescindível o conhecimento e a validação de métodos laboratoriais para que estes possam fornecer resultados fidedignos de detecção e identificação dos mecanismos de resistências, contribuindo de maneira eficaz na redução dos índices de morbidade e mortalidade.

Este estudo teve por objetivo validar o teste de inibição por APBA como método de triagem para pesquisa fenotípica de cepas produtoras de enzimas betalactamases tipo KPC, comparando os resultados obtidos por esse teste com os dos testes confirmatórios por reação em cadeia da polimerase (PCR).

\section{Materiais e métodos}

\section{Critérios de inclusão}

\section{Amostras positivas}

Trinta e seis cepas de K. pneumoniae, confirmadas como produtoras de betalactamase do tipo KPC por PCR, utilizando os primers: KPC A - sequência $5^{\prime}-3^{\prime}$ : CTCTCTTGTCTCTCATGGCC; e KPC B - sequência $5^{\prime}-3^{\prime}$ : CCTCGCTGTGCTTGTCATCC.

\section{Amostras negativas}

Doze cepas de bactérias, não produtoras de KPC, confirmadas por PCR. Foram incluídas outras espécies além de K. pneumoniae.

\section{Microrganismos}

Foram utilizados 48 isolados clínicos da família Enterobacteriaceae, previamente identificadas pelo sistema Vitek $^{\circledR} 2$ (bioMéuriex), cedidas pelo Laboratório Central do Estado do Paraná (LACEN). Entre as 48 cepas, 36 eram K. pneumoniae, confirmadas como produtoras de enzima KPC por PCR; dessas 36 cepas, 12 também eram produtoras de betalactamases de amplo espectro (ESBL), identificadas pelo sistema automatizado Vitek ${ }^{\circledast} 2$ (bioMéuriex). Entre as 12 cepas restantes que foram confirmadas negativas para a produção de KPC por PCR, seis eram K. pneumoniae; duas, Enterobacter cloacae; uma, Escherichia coli; e uma, Enterobacter aerogenes. As cepas mantidas em caldo brain heart infusion $(\mathrm{BHI})+$ glicerol a $-70^{\circ} \mathrm{C}$ foram previamente reativadas por repique em Ágar MacConkey e incubadas a $36^{\circ} \mathrm{C}$ por 24 horas, antes da realização do teste de inibição pelo APBA.

\section{Amplificação por PCR}

Todas as cepas foram previamente confirmadas molecularmente para a presença ou não do gene $b / a_{\mathrm{KPC}}$. A PCR foi feita por método in house. Primeiramente, preparou-se uma solução master mix, a qual continha os primers forward e reverse, sendo KPC A: sequência $5^{\prime}-3^{\prime}$ : CTCTCTTCTCTCTCATGGCC, KPC B: sequência 5' -3’: CCTCGCTGTGCTTGTCATCC; Taq polimerase (Taq ácido desoxirribonucleico [DNA] polymerase recombinante, invitrogen), dNTPs, $\mathrm{MgCl}_{2}$ e DNA previamente extraído conforme explanado por Naas et al. ${ }^{(10)}$. Os tubos de reação foram levados ao termociclador, no qual foi realizada a amplificação do DNA com desnaturação inicial de $94^{\circ} \mathrm{C}$ por 5 minutos, seguido de 35 ciclos de desnaturação a $94^{\circ} \mathrm{C}$ por 45 segundos, anelamento do primer a $65^{\circ} \mathrm{C}$ por 45 segundos e extensão a $72^{\circ} \mathrm{C}$ por 45 segundos, além de uma extensão final de $72^{\circ} \mathrm{C}$ por 7 minutos. O gene pesquisado foi o $b / a_{\mathrm{KPC}}$ e o resultado se deu por meio da presença de uma banda específica em eletroforese em gel de agarose $2 \%$. 


\section{Teste de inibição pelo ácido borônico para identificação fenotípica de KPC}

\section{Preparação dos discos}

Discos de antibióticos contendo $30 \mu \mathrm{g}$ de meropenem, imipenem e ertapenem (Oxoid ${ }^{\circledR}$ ) foram impregnados com solução aquosa de APBA (Sigma Aldrich ${ }^{\circledR}$ ) $50 \mathrm{mg} / \mathrm{ml}$, que foi adicionada nos volumes de $6 \mu \mathrm{l}, 8 \mu \mathrm{l}$ e $12 \mu \mathrm{l}$, gerando três concentrações finais de APBA $(300,400$ e $600 \mu \mathrm{g}$, respectivamente) para cada antibiótico. Controle dos discos sem APBA também foram realizados. Os discos foram deixados para secar por 30 minutos e armazenados a $-20^{\circ} \mathrm{C}$ por até três semanas ${ }^{(5)}$.

\section{Controles positivo e negativo}

Foi utilizada como controle negativo a cepa de Klebsiella pneumoniae ATCC 13883, sensível aos carbapenêmicos. Como controle positivo, utilizou-se cepa de Klebsiella pneumoniae, $\mathrm{n}^{\circ}$ 2470, cedida pelo Instituto Adolfo Lutz (IAL), confirmada como produtora de KPC por PCR. Os testes para os controles foram realizados em triplicata.

\section{Ensaio de sinergismo}

O teste de inibição pelo APBA foi realizado de acordo com DOI et al.(6). Para cada cepa, preparou-se uma suspensão direta em salina $0,85 \%$ a partir de colônias isoladas com 24 horas de crescimento, e a turvação da suspensão foi ajustada de acordo com o tubo 0,5 de Mac Farland, utilizando-se um densitômetro (Densimat ${ }^{\oplus}$ - bioMérieux) com comprimento de onda de $625 \mathrm{~nm}$, o que gera uma suspensão de

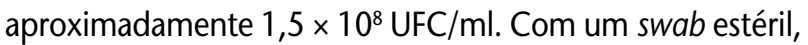
a suspensão foi inoculada na superfície da placa de Ágar Muller-Hinton. Após, os discos foram colocados sob a superficie da placa inoculada e estas foram incubadas a $35 \pm$ $2^{\circ} \mathrm{C}$ por 18-24 horas.

Os resultados foram obtidos pela leitura dos halos de inibição obtidos sem e com a adição da solução de APBA. Quando o halo de inibição ao redor do disco contendo o antibiótico mais APBA for maior ou igual a $5 \mathrm{~mm}$ que o halo do disco sem a solução de APBA, a prova será considerada positiva para a produção de $\mathrm{KPC}^{(40)}$. Os resultados foram ainda analisados sob a perspectiva de novo ponto de corte ( $3 \mathrm{~mm})$, na tentativa de otimizar a sensibilidade do teste.

\section{Parâmetros de avaliação e validação do teste}

Os resultados obtidos foram comparados com os resultados fornecidos pelo teste molecular. Determinou-se, ainda, a sensibilidade e a especificidade para cada concentração de APBA e para cada antibiótico, de acordo com a análise sugerida por Ilstrup ${ }^{(7)}$. Diferenças estatísticas entre os resultados obtidos com as diferentes concentrações de APBA e antibióticos foram avaliadas por análise de variância (ANOVA), com nível de significância 0,05.

\section{Resultados}

Para as 48 amostras testadas, como critério para avaliação dos resultados foi considerada amostra verdadeira positiva/negativa aquela obtida por PCR. Na Tabela 1, pode-se observar que, entre as 36 cepas positivas para a presença da enzima KPC por PCR, apenas 11, 8 e 12 foram positivas pelo método proposto, utilizando meropenem com adição de 300,400 e $600 \mu \mathrm{g}$, respectivamente. Utilizando o antibiótico ertapenem com a adição de 300, 400 e $600 \mu \mathrm{g}$, apenas 15, 12 e 18 foram positivas, respectivamente. Em relação ao imipenem, entre as 36 cepas positivas pelo PCR, nenhuma se apresentou positiva com a utilização desse antibiótico. No entanto, para as cepas negativas pelo PCR para a presença de KPC, o método proposto forneceu resultado negativo para todas as 12 cepas molecularmente negativas com os três antibióticos testados.

Observam-se na Tabela 2 os resultados obtidos com ponto de corte de $3 \mathrm{~mm}$. Dessa forma, das 36 amostras verdadeiras positivas, meropenem nas concentrações de APBA 300, 400 e $600 \mu \mathrm{g}$, foi capaz de detectar 25, 21 e 26 amostras, respectivamente, enquanto ertapenem foi capaz de detectar 28, 31 e 31, respectivamente. Imipenem, todavia, detectou apenas duas amostras, quando associado ao APBA $300 \mu \mathrm{g}$. Quando foram utilizadas concentrações de 400 e $600 \mu \mathrm{g}$, este não detectou nenhuma amostra positiva. Quanto à concordância entre os métodos nas amostras negativas, meropenem e imipenem forneceram resultados negativos para todas as amostras negativas por PCR, contudo, observaram-se dois resultados falso-positivos quando se utilizou ertapenem em associação com APBA 300 e $400 \mu \mathrm{g}$, e quatro falso-positivos com APBA $600 \mu \mathrm{g}$.

Com o ponto de corte de $5 \mathrm{~mm}$, o ertapenem apresentou sensibilidade de $50 \%, 41,67 \%$ e $33,33 \%$ quando associado a $600 \mu \mathrm{g}, 300 \mu \mathrm{g}$ e $400 \mu \mathrm{g}$, respectivamente. O meropenem apresentou sensibilidade de 33,33\% com $600 \mu \mathrm{g}$ de APBA, 30,56\% com $300 \mu$ g e 2,22\% com $400 \mu \mathrm{g}$. Para o antibiótico imipenem, a sensibilidade foi de $0 \%$ para todas as concentrações de APBA testadas (Figura 1). Com o ponto de corte de $3 \mathrm{~mm}$, ertapenem apresentou sensibilidade de $86,11 \%$ quando utilizado em associação com $400 \mathrm{e}$ 
Tabela 1 cepas produtoras de KPC

\begin{tabular}{|c|c|c|c|c|}
\hline \multirow{2}{*}{ Antibiótico } & \multirow{2}{*}{ Concentração de APBA } & \multirow{2}{*}{ Teste de inibição pelo APBA } & \multicolumn{2}{|c|}{ PCR } \\
\hline & & & Positiva & Negativa \\
\hline \multirow{6}{*}{ Meropenem } & \multirow{2}{*}{$300 \mu \mathrm{g}$} & Positivo & 11 & 0 \\
\hline & & Negativo & 25 & 12 \\
\hline & \multirow{2}{*}{$400 \mu g$} & Positivo & 8 & 0 \\
\hline & & Negativo & 28 & 12 \\
\hline & \multirow{2}{*}{$600 \mu g$} & Positivo & 12 & 0 \\
\hline & & Negativo & 24 & 12 \\
\hline \multirow{6}{*}{ Ertapenem } & \multirow{2}{*}{$300 \mu g$} & Positivo & 15 & 0 \\
\hline & & Negativo & 21 & 12 \\
\hline & \multirow{2}{*}{$400 \mu g$} & Positivo & 12 & 0 \\
\hline & & Negativo & 24 & 12 \\
\hline & \multirow{2}{*}{$600 \mu g$} & Positivo & 18 & 0 \\
\hline & & Negativo & 18 & 12 \\
\hline \multirow{6}{*}{ Imipenem } & \multirow{2}{*}{$300 \mu g$} & Positivo & 0 & 0 \\
\hline & & Negativo & 36 & 12 \\
\hline & \multirow{2}{*}{$400 \mu g$} & Positivo & 0 & 0 \\
\hline & & Negativo & 36 & 12 \\
\hline & \multirow{2}{*}{$600 \mu g$} & Positivo & 0 & 0 \\
\hline & & Negativo & 36 & 12 \\
\hline
\end{tabular}

Comparativo dos resultados obtidos entre meropenem, ertapenem e imipenem em diferentes concentrações de APBA e teste molecular (PCR) - (ponto de corte de $5 \mathrm{~mm}$ ) para a detecção de

\section{A cepas produtoras de KPC}

Concentração de APBA

APBA: 3-aminofenil ácido borônico; PCR: reação em cadeia da polimerase; KPC: Klebsiella pneumoniae carbapenemase.

\section{Comparativo dos resultados obtidos entre meropenem, ertapenem e imipenem em diferentes \\ concentrações de APBA e teste molecular (PCR) - (ponto de corte de $3 \mathrm{~mm}$ ) para a detecção de}

Tabela 2 cepas produtoras de KPC

\begin{tabular}{|c|c|c|c|c|}
\hline \multirow{2}{*}{ Antibiótico } & \multirow{2}{*}{ Concentração de APBA } & \multirow{2}{*}{ Teste de inibição pelo APBA } & \multicolumn{2}{|c|}{ PCR } \\
\hline & & & Positiva & Negativa \\
\hline \multirow{6}{*}{ Meropenem } & \multirow{2}{*}{$300 \mu g$} & Positivo & 25 & 0 \\
\hline & & Negativo & 11 & 12 \\
\hline & \multirow{2}{*}{$400 \mu g$} & Positivo & 21 & 0 \\
\hline & & Negativo & 15 & 12 \\
\hline & \multirow{2}{*}{$600 \mu g$} & Positivo & 26 & 0 \\
\hline & & Negativo & 10 & 12 \\
\hline \multirow{6}{*}{ Ertapenem } & \multirow{2}{*}{$300 \mu g$} & Positivo & 28 & 2 \\
\hline & & Negativo & 8 & 10 \\
\hline & \multirow{2}{*}{$400 \mu g$} & Positivo & 31 & 2 \\
\hline & & Negativo & 5 & 10 \\
\hline & \multirow{2}{*}{$600 \mu \mathrm{g}$} & Positivo & 31 & 4 \\
\hline & & Negativo & 5 & 8 \\
\hline \multirow{6}{*}{ Imipenem } & \multirow{2}{*}{$300 \mu g$} & Positivo & 2 & 0 \\
\hline & & Negativo & 34 & 12 \\
\hline & \multirow{2}{*}{$400 \mu g$} & Positivo & 0 & 0 \\
\hline & & Negativo & 36 & 12 \\
\hline & \multirow{2}{*}{$600 \mu g$} & Positivo & 0 & 0 \\
\hline & & Negativo & 36 & 12 \\
\hline
\end{tabular}

APBA: 3-aminofenil ácido borônico; PCR: reação em cadeia da polimerase; KPC: Klebsiella pneumoniae carbapenemase. 


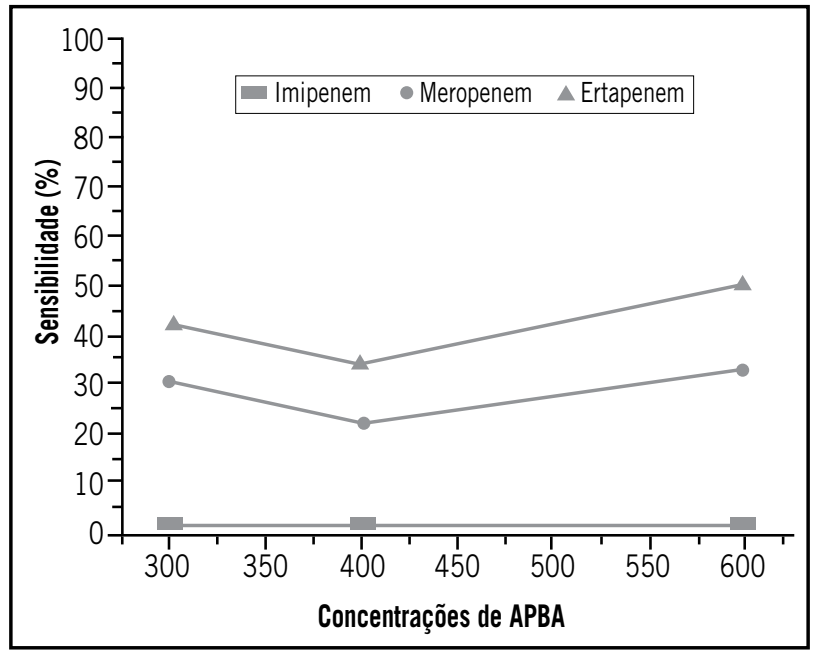

Figura 1 - Sensibilidade (\%) do teste de inibição pelo APBA para cada antibiótico e concentração de APBA (ponto de corte $=5 \mathrm{~mm}$ ) para a detecção de cepas produtoras de KPC

APBA: 3-aminofenil ácido borônico; KPC: Klebsiella pneumoniae carbapenemase.

$600 \mu \mathrm{g}$ de APBA e de $77,78 \%$ com $300 \mu \mathrm{g}$. Para meropenem, a sensibilidade foi de $69,44 \%, 58,33 \%$ e $72,22 \%$ ,respectivamente, com as concentrações de 300, 400 e 600 $\mu \mathrm{g}$ de APBA. Imipenem mostrou sensibilidade de apenas $5,56 \%$ quando associado a $300 \mu$ de APBA (Figura 2).

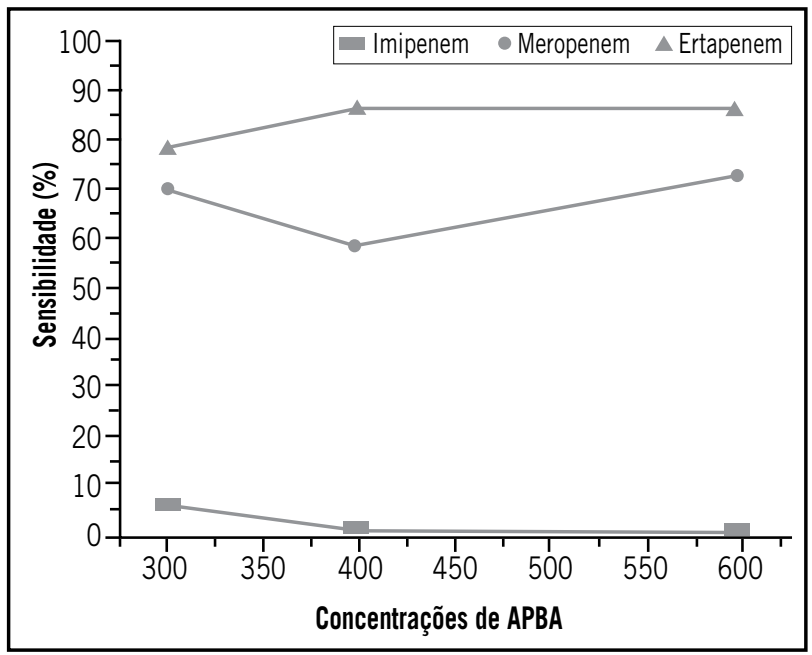

Figura 2 - Sensibilidade (\%) do teste de inibição pelo APBA para cada antibiótico e concentração de APBA (ponto de corte $=3 \mathrm{~mm}$ ) para a detecção de cepas produtoras de KPC

APBA: 3-aminofenil ácido borônico; KPC: Klebsiella pneumoniae carbapenemase

$\mathrm{Na}$ Figura 3, os dados são expressos como média \pm erro padrão (EP) dos halos de inibição para os três antibióticos nas três concentrações de APBA. Imipenem $0 \mu \mathrm{g}$ APBA $(16,36 \pm 0,691) ; 300 \mu \mathrm{g}(12,44 \pm 0,75) ; 400 \mu \mathrm{g}(6,89 \pm$ $0,385) ; 600 \mu \mathrm{g}(6 \pm 0)$. Meropenem $0 \mu \mathrm{g}$ APBA $(12,69 \pm$ $0,761) ; 300 \mu \mathrm{g}(15,97 \pm 0,613) ; 400 \mu \mathrm{g}(15,69 \pm 0,671)$; $600 \mu \mathrm{g}(16,03 \pm 0,705)$. Ertapenem $0 \mu \mathrm{g}$ APBA $(8,64 \pm$
0,645); $300 \mu \mathrm{g}(12,78 \pm 0,561) ; 400 \mu \mathrm{g}(12,64 \pm 0,626) ;$ $600 \mu \mathrm{g}(13,11 \pm 0,623)$. Observa-se que a adição de APBA ao antibiótico imipenem ocasionou diminuição dos halos de inibição, enquanto para o ertapenem e meropenem, os halos de inibição se mantiveram os mesmos ou aumentaram de tamanho com a adição de APBA. A diferença entre os halos de inibição observada entre meropenem e ertapenem apresenta significância estatística com $p<0,001,<0,001$, $<0,01$ para as concentrações 300,400 e $600 \mu \mathrm{g}$, respectivamente. Entretanto, diferenças entre as concentrações de APBA, tanto para meropenem quanto para ertapenem, só foram significativas entre a concentração $0 \mu \mathrm{g}$ e as demais (meropenem, $p<0,05$ e ertapenem, $p<0,01$ ), não sendo, portanto, significativa a diferença entre as concentrações 300, 400 e $600 \mu \mathrm{g}$ para os dois antibióticos (meropenem, $p=0,983 ; 1,000 ; 0,983$ ) (ertapenem, $p=0,993 ; 0,970$; $0,892)$.

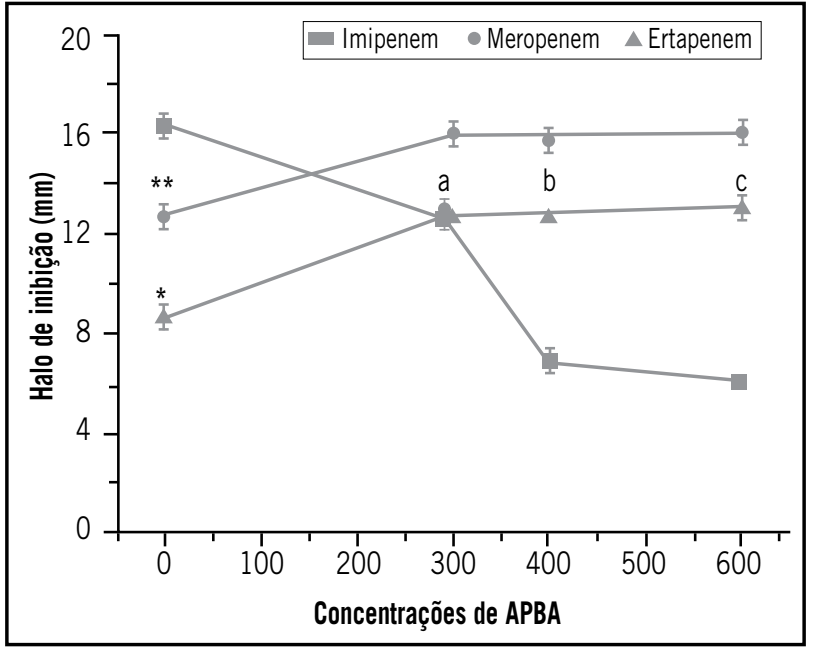

Figura 3 - Comportamento dos halos de inibição para cada antibiótico de acordo com a concentração de APBA

* Diferença estatística entre as concentrações $0 \mu \mathrm{g}$ de demais APBA (300, 400 e $600 \mu g)$ para antibiótico ertapenem $(p<0,01)$.

** Diferença estatística entre as concentrações $0 \mu \mathrm{g}$ de demais APBA $(300,400 \mathrm{e}$ $600 \mu g)$ para antibiótico meropenem $(\mathrm{p}<0,05)$.

(a) diferença estatística entre os antibióticos meropenem e ertapenem na concentração de $300 \mu \mathrm{g}$ de APBA ( $\mathrm{p}<0,001)$; (b) diferença estatística entre os antibióticos meropenem e ertapenem na concentração de $400 \mu g$ de APBA ( $p<0,001)$; (c) diferença estatística entre os antibióticos meropenem e ertapenem na concentração de $600 \mu g$ de APBA ( $\mathrm{p}<0,01)$.

\section{Discussão}

O número de casos de resistência aos carbapenêmicos tem aumentado em vários centros brasileiros e no mundo todo, o que tem se tornado grande motivo de preocupação, sobretudo no ambiente hospitalar ${ }^{(3,11,15)}$. 
Diante disso e da limitação terapêutica existente, a detecção correta desses mecanismos de resistência se torna o passo fundamental no controle da disseminação. Diversas técnicas moleculares têm oferecido ótimo desempenho na detecção dessas enzimas, no entanto, são limitadas por serem de alto custo. O teste de Hodge modificado é uma técnica menos onerosa, porém, sua interpretação requer alguma experiência; ademais, o teste pode apresentar resultados falso-positivos ${ }^{(12,13)}$.

De acordo com relatos que avaliaram a eficácia do teste de inibição pelo ácido borônico na deteç̧ão de enzimas betalactamases, pode-se observar um excelente desempenho do teste para este fim, já que exibiu alta sensibilidade e especificidade na maioria dos estudos realizados ${ }^{(4,17)}$. Em estudo conduzido por $\mathrm{DOI}^{(6)}$ que avaliou a atividade inibitória do ácido aminofenilborônico, impregnando discos de antibióticos carbapenêmicos, demonstrou concordância que excede $90 \%$, sendo o ertapenem e meropenem substratos que apresentaram maior sensibilidade, em comparação ao imipenem. No entanto, o presente estudo apresentou baixa sensibilidade do teste utilizando APBA na detecção de enzimas KPC em três diferentes concentrações de APBA e em três diferentes substratos. Isso também foi observado em um estudo realizado por Su et al.(16), que utilizou duas diferentes formulações de ácido borônico (APB e APBA) e a mesma técnica, na qual discos de cefotaxima e ceftazidima impregnados com solução de APB e APBA foram utilizados para detecção de enzimas do tipo ampC. Uma hipótese seria que os diferentes percentuais de sensibilidade encontrados entre este estudo e os outros para detecção dessas enzimas podem ter ocorrido devido a diferentes formulações de ácido borônico utilizadas. Outra pesquisa também sugere que a capacidade de detecção das enzimas KPC por APBA é menor quando comparada com $P B A^{(13)}$. Diante disso, faz-se necessário mais estudos para aperfeiçoar e padronizar a técnica a fim de otimizar a detecção desses mecanismos de resistência, bem como melhorar a sensibilidade e a especificidade dos testes, sobretudo na detecção de enzimas do tipo KPC.

A diminuição do ponto de corte para $3 \mathrm{~mm}$ foi sugerida com o intuito de otimizar os resultados, buscando um aumento na sensibilidade do teste, sem diminuir a especificidade. Sob esse novo aspecto, pode-se observar que houve aumento significativo na sensibilidade, que foi de $50 \%$ para $86,11 \%$ para o ertapenem com APBA $400 \mu \mathrm{g}$. Já a especificidade, para o mesmo antibiótico e a mesma concentração, apresentou uma ligeira queda, passando para 83,33\%. Como o objetivo do teste com APBA é ser implantado para triagem, a diminuição do ponto de corte mostrou-se eficaz, pois aumenta consideravelmente a sensibilidade. Já a diminuição da especificidade não compromete sua eficácia, uma vez que esses resultados precisam ser confirmados por métodos moleculares.

\section{Conclusão}

Diante deste estudo, observa-se que o teste de inibição pelo ácido borônico mostra-se como um potencial teste de triagem para deteç̧ão da enzima KPC. Contudo, a validação pretendida neste trabalho não foi possível, o que aponta a necessidade de novas pesquisas que visem a padronização da técnica, com a escolha da melhor formulação de ácido borônico, determinação da estabilidade da solução e dos antibióticos quando submetidos ao armazenamento, bem como determinação dos pontos de corte utilizados para interpretação do teste, e até mesmo avaliação de outros métodos, como o de disco aproximação. Todo esforço no sentido de aperfeiçoar a técnica é importante, pois torna-se necessária a implantação de novos testes na rotina laboratorial que sejam de fácil execução e que permitam a identificação correta de surtos, sobretudo em ambientes hospitalares, pois eles têm sofrido com o crescente aumento nos índices de morbidade e mortalidade relacionadas com esse mecanismo de resistência.

\section{Agradecimentos}

Ao LACEN-PR, à equipe do setor da Bacteriologia Geral, Karin Obladen, Thales Wuicik e Marcella Duarte, e ao Luiz Augusto da Silva, pelo auxílio estatístico.

\section{Referências}

1. ALBA, J. et al. Kinetics study of KPC-3, a plasmid-encoded class A carbapenem-hydrolyzing lactamase. Antimicrob Agents Chemother, v. 49, p. 4760-2, 2005.
2. BRATU, S. et al. Detection of KPC carbapenem-hydrolyzing enzymes in Enterobacter spp. Antimicrob Agents Chemother, v. 49, p. 776-8, 2005. 
3. BRATU, S. et al. Rapid spread of carbapenemresistant Klebsiella pneumoniae in New York city: a new threat to our antibiotic armamentarium. Arch Intern Med, v. 165 , p. $1430-5,2005$.

4. BRENWALD, N. P. et al. Disc methods for detecting AmpC $\beta$-lactamase-producing clinical isolates of Escherichia coli and Klebsiella pneumoniae. J Antimicrob Chemother, v. 56, p. 600-1, 2005.

5. COUDRON, P. E. Inhibitor-based methods for detection of plasmid-mediated AmpC $\beta$-lactamases in Klebsiella spp., Escherichia coli, and Proteus mirabilis. J Clin Microbiol, v. 43, p. 4163-7, 2005.

6. DOI, Y. et al. Simple disk-based method for detection of Klebsiella pneumoniae carbapenemase-type-lactamase by use of a boronic acid compound. J Clinl Microbiol, v. 46, n. 12, p. 4083-6, 2008.

7. ILSTRUP, D. M. Statistical methods in microbiology. Clin Microbiol Rev, v. 3, p. 219-26, 1990.

8. MARCHAIM, D. et al. Isolation of imipenemresistant Enterobacter species: emergence of KPC-2 carbapenemase, molecular characterization, epidemiology, and outcomes. Antimicrob Agents Chemother, v. 52, p. 1413-8, 2008.

9. MIRIAGOU, V. et al. Imipenem resistance in a Salmonella clinical strain due to plasmid-mediated class $A$ carbapenemase KPC-2. Antimicrob Agents Chemother, v. 47, p. 1297-1300, 2003.

10. NAAS, T. et al. Plasmid-mediated carbapenem-hydrolyzing $\beta$-lactamase KPC in a Klebsiella pneumoniae isolate from France. Antimicrob Agents Chemother, v. 49, p. 4423-4, 2005.

11. NORDMANN, P.; CUZON G.; NAAS, T. The real threat of Klebsiella pneumoniae carbapenemase-producing bacteria. Lancet Infect Dis, v. 9, p. 228-36, 2009.
12. PASTERAN, F. et al. Controlling false-positive results obtained with the hodge and masuda assays for detection of alass A carbapenemase in species of Enterobacteriaceae by incorporating boronic acid. J Clin Microbiol, v. 48, n. 4, p. 1323-32, 2010.

13. POURNARAS, S.; POULOU, A.; TSAKRIS, A. Inhibitor-based methods for the detection of KPC carbapenemasesproducing Esterobatereaceae in clinical practice by using boronic acid compounds. J Antimicrob Chemother, 2010.

14. QUEENAN, A. M.; BUSH, K. Carbapenemases: the versatile $\beta$-lactamases. Clinical Microbiology Review, v. 20, p. 440-58, 2007.

15. SMITH M. E. et al. Plasmid-mediated, carbapenemhydrolysing $\beta$-lactamase, KPC-2, in Klebsiella pneumonia isolates. J Antimicrob Chemother, v. 51, p. 711-4, 2003

16. SU, W. Y.; GOTTLIEB, T.; MERLINO, J. Optimal phenotypic testing of ampC beta-lactamases using boronic acid solutions. Eur J Clin Microbiol Infect Dis, 2011.

17. TENOVER, F. C. et al. Carbapenem resistance in Klebsiella pneumoniae not detected by automated susceptibility testing. Emerg Infect Dis, v. 12, p. 1209-13, 2006.

18. TSAKRIS, A. et al. Evaluation of boronic acid disk tests for differentiating KPC-possessing Klebsiella pneumoniae isolates in the clinical laboratory. J Clin Microbiol, v. 47, p. 362-7, 2009.

19. VILLEGAS, M. V. et al. First identification of Pseudomonas aeruginosa isolates producing a KPC-type carbapenemhydrolyzing-lactamase. Antimicrob Agents Chemother, v. 51, p. 1553-5, 2007.

20. YIGIT, H. et al. Carbapenem-resistant strain of Klebsiella oxytoca harboring carbapenem-hydrolyzing $\beta$ - lactamase KPC-2. Antimicrob Agents Chemother, v. 47, p. 3881-9, 2003. 